International Journal of Scientific Research in Science, Engineering and Technology

Print ISSN: 2395-1990 | Online ISSN : 2394-4099 (www.ijsrset.com)

doi : https://doi.org/10.32628/IJSRSET2183130

\title{
Covid-19 Detection Using AI
}

\author{
Shashank Mishra, Himanshu Kumar Shukla, Rajiv Singh, Vivek Pandey, Shubham Sagar, Yasasvi Singh \\ Department of Computer Science and Engineering Babu Banarasi Das Institute of Technology and \\ Management, Lucknow, Uttar Pradesh , India
}

Article Info
Volume 8, Issue 3
Page Number: 561-566

Publication Issue :

May-June-2021

\section{Article History}

Accepted : 20 June 2021

Published: 30 June 2021

\section{ABSTRACT}

The sudden increase in COVID-19 patients is a major shock to our global health care systems. With limited availability of test kits, it is not possible for all patients with respiratory infections to be tested using RT-PCR. Testing also takes a long time, with limited sensitivity. The detection of COVID-19 infections on Chest X-Ray can help isolate patients at high risk while awaiting test results. X-Ray machines are already available in many health care systems, and with many modern X-Ray systems already installed on the computer, there is no travel time involved in the samples. In this work we propose the use of chest X-Ray to prioritize the selection of patients for further RT-PCR testing. This can be useful in a hospital setting where current systems have difficulty deciding whether to keep the patient in the ward with other patients or isolate them from COVID-19 areas. It may also be helpful in identifying patients with high risk of COVID with false positive RT-PCR that will require repeated testing. In addition, we recommend the use of modern AI techniques to detect COVID-19 patients who use X-Ray imaging in an automated manner, especially in areas where radiologists are not available, and help make the proposed diagnostic technology easier. Introducing the CovidAID: COVID-19 AI Detector, a model based on a deep neural network of screening patients for proper diagnosis. In a publicly available covid-chest $\mathrm{x}$-ray-dataset [2], our model provides $90.5 \%$ accuracy with $100 \%$ sensitivity (remember) to COVID19 infection. We are greatly improving the results of Covid-Net [10] on the same database.

Keywords : Deep learning, CNN Algorithm Chest X-Ray Neural Network

\section{INTRODUCTION}

The sudden increase in the number of patients with COVID-19, a new respiratory virus, has put an unprecedented burden on health care systems worldwide. In many lands, health care systems are in crisis. There there are limited diagnostic kits, limited hospital beds for admission to such patients, limited medical equipment (PPE) for health care workers and limited ventilators. It is therefore important to differentiate which patients with severe respiratory infections (SARI) may have COVID-19 infection in 
order to make the best use of the prescribed resources. In this work we propose the use of chest X-Ray to detect COVID-19 infection in patients showing SARI symptoms. Using our tool one can classify X-Ray given in one of four classes: common, bacterial pneumonia, viral pneumonia, and covid pneumonia. The use of X-Ray has several advantages over conventional diagnostic tests: 1 . X-ray imaging is more widespread and more expensive than conventional diagnostic tests. 2. X-Ray digital image transfer does not require transfer from location to location analysis, which makes the diagnostic process much faster. 3. Unlike CT Scans, portable X-Ray machines also enable testing within the isolation ward itself, thus reducing the need for additional Personal Protective Equipment (PPE), a scarce and very important resource in this situation. It also reduces the risk of infection with patients in the hospital. The main contribution of this work is to propose a model based on a deep network to obtain accurate diagnosis of COVID-19 infection from X-Ray chest images of patients. Radiographs in the present case in most cases are translated by non-radiologists. In addition, if you are given a new virus, most radiologists themselves may not know all the nuances of the infection, and may lack sufficient expertise to make a more accurate diagnosis. So this automated tool can serve as a guide for those leading this study. We would also like to emphasize that we do not recommend the use of the proposed model as one of the standard tests for diagnosing COVID19 infection, but as a diagnostic tool to determine the suitability of a patient with SARI to perform COVID-19 infection tests. Percentage of patients diagnosed, mortal, low and critical were defined as $86 \%, 14 \%, 98 \%$ and $2 \%$ respectively.

\section{RESEARCH GAP}

Recently, several studies have investigated the therapeutic images of COVID-19 using a variety of indepth study methods. Wang et al. [25] presented an open source study model and observed a large benchmark database called COVID $\mathrm{x}$ with 13,975 patient chest X-ray images to test COVID-19. This model not only verified the larger details of the sensitive features of COVID-19 but also extracted relevant information from the experimental images. Their study produced $93.3 \%$ accuracy over the COVID x database. Maghdid et al. [3] used a deep convolution neural network (CNN) and transmitted learning using Alex Net on X-ray and CT scan image datasets. They indicated 94\% accuracy (88\% detail) for CNN and $98 \%$ for Alex Net (96\% detail). Subsequently, Ali Narin et al. [21] analyzed chest XRay images using three CNN models such as ResNet50, InceptionV3 and InceptionResNetV2 for automatic COVID-19 detection. Their study showed a very high accuracy of $98 \%$ using 5-fold verification. Abbas et al. [1] CNN's proposed DeTraC model aims to provide a solution by transferring information from standard object recognition to domain-specific functions. Their algorithm showed 95.55\% accuracy (91.87\% accuracy, and 93.36\% accuracy). Apostolopoulos and Mpesiana [3] used reading transfers using CNNs in a small medical image database. Their database contained $1427 \mathrm{X}$-ray images including 224 COVID-19 certified images. They showed the best accuracy of $96.78 \%$, sensitivity of $98.66 \%$, and specificity of $96.46 \%$ respectively. Hemdan, Shouman and Karar [11] proposed COVIDX-Net including seven deep CNN structures that investigated 50 chest X-ray images for 25 COVID-19 cases. Their algorithm produced 90\% accuracy and 91\% F-points. Subsequently, Kobahi, Agarwal and Soltanalian [13] proposed a slightly supervised reading method based on an autoencoder that ensures an average accuracy of $93.5 \%$. Li et al. [15] provided a weight-based mobile app with COVID-MobileXpert. Sound chest X-rays can be used in COVID-19 tests. Therefore, both ShuffleNetV2 and MobileNetV2 produced AUROC high prices of $94 \%$ and $94.30 \%$ respectively. Minaee et al. [20] proposed an in-depth reading framework based on 5000 images called COVID-Xray-5k where they used ResNet18, 
Available under license from CC-BY-NC-ND 4.0 International. (unconfirmed by peer review) by author / sponsor, who licensed MedRxiv to display fraud. doRxiv preprint doi: https://doi.org/10.1101/2020.06.07.20124594; this version was posted June 8, 2020. Copyright holder of this preprint 4 Khair Ahammed et al. ResNet50, SqueezeNet and Densenet-121, also demanded 97.5\% sensitivity and $90 \%$ specificity on average. Alom et al. [2] used in-depth study methods to identify COVID19 patients using X-ray images and CT scans. Their algorithm found the end of COVID-19 and local infected areas using in-depth learning methods. They also used CNN's Inception Residual Recurrent with Transfer Learning and received $84.67 \%$ accuracy in $\mathrm{X}$-ray imaging and $98.78 \%$ accuracy in CT imaging. Finally, Zhang et al. [26] pointed to the creation of an in-depth abstract finding model that works on a database of Xray chest images. Their separator gained $96 \%$ sensitivity to positive COVID-19 cases and $70.65 \%$ specificity of negative COVID-19 cases.

\section{METHOD}

We suggest using a deep convolution neural network specialized to find something and a new database with images of COVID-19 and pneumonia. Both are publicly available on GitHub [14] and Kaggle [15]respectively. The Chest X-ray or CT images available on GitHub for COVID-19 cases. Created by integrating medical images from websites and existing public publications. This database contains $204 \mathrm{X}$-ray images of COVID-19. Kaggle's database, on the other hand, has been challenged for pneumonia. The pictures have binding boxes around the infected areas. Samples outside the binding boxes are nutritious and contain clear evidence of pneumonia. Samples with binding boxes show evidence of pneumonia. We are proposing a new database by combining images of COVID-19 and pneumonia to find a wide and varied one. The fact that they have pneumonia images in the training database assumes a greater benefit, due to the common pneumonia and COVID-19 may have similar effects on chest $\mathrm{X}$-ray images. This combination of data allows finding a robuster model that can better differentiate between those diseases. Another benefit of this merger is the fact that the railway database has been expanded, as COVID-19 photographs are not available at the time of writing. This combination does not increase the setting of the COVID-19 image but improves the quality of detection due to the similarity between pneumonia and COVID-19. The train with pneumonia images provides additional information to the model so that it does not confuse COVID-19 with pneumonia; it is more efficient and more stable in diagnosing diseases. We separated the images into train and test images, we separated all the data in a balanced way, which means that all samples of each class in the training sets were well-balanced, to avoid the effects of bias. For this purpose, although we have a large number of pneumonia and standard images, we are composing 1500 images.

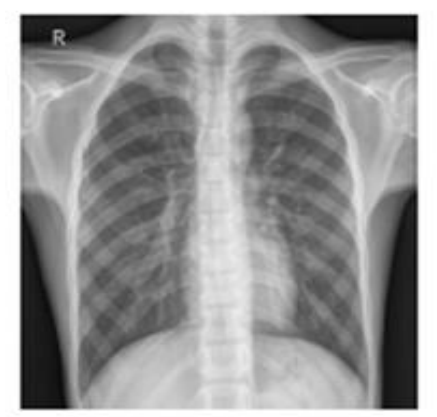

(a) Normal Lung

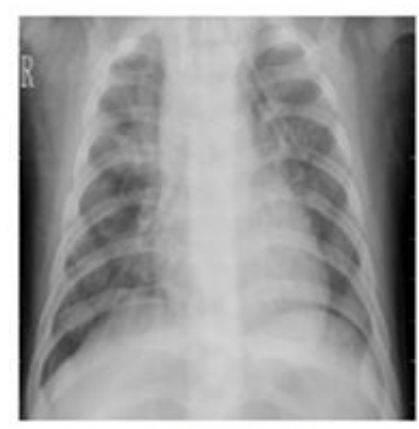

(c) Viral

Pneumonia

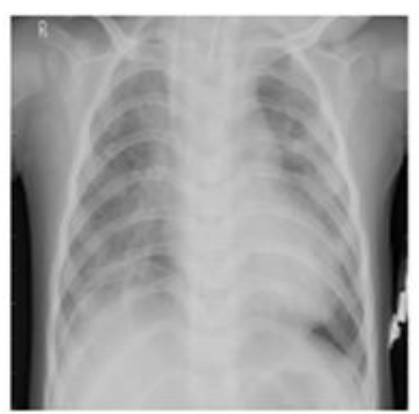

(b) Bacterial

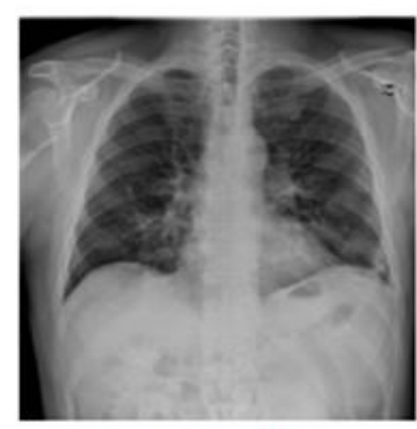

(d) COVID-19 Pneumonia

Figure 1 : Category of Lungs Image 
We build the database as follows: we select 104 COVID-19 images, 205 lung images and 204 pneumonia training images, 100 COVID-19 pads, 444 pneumonia and 443 pneumonia images to be tested. In Kaggle's database there are many examples of pneumonia and typical images, but we select only 205 for the purpose of having a balanced database. In the experimental phase, we included additional pneumonia and standard images to show the firmness of the model without providing a positive effect on the detection of COVID-19. In summary, we use a train set of 513 images and a test set of 887 images in total. In the next step, the training images are labeled using an XML annotation file based on the Pascal VOC format [16].

\section{IMPLEMENTATION}

The choice of properties used is based on the positive results obtained by CNN in the artistic performance of the COVID-19 image class, as well as the positive results obtained from other similar works with this type of properties [11] [12] [13]. We used the same network configuration as suggested in [17], based on the Single Shot Multibox Detector (SSD). This structure is designed to detect objects in images using a single deep neural network. This method creates no space for the release of binding boxes in a set of default boxes in addition to the rating of various features and scales by the location of the feature map. During forecasting, the network generates the availability scores for each item category in each default box and generates box adjustments to better match the composition of the item. Additionally, the network includes predictions from multi-featured maps with different resolutions for naturally handling objects of different sizes. Test results on distinct distinctive data sets ensure that the SSD has comparable accuracy with methods that use more than one format to detect objects much faster, while providing an integrated framework for training and inclination. Compared to other single-stage methods, the SSD has a much better accuracy, or with a smaller input image size [17].

We are using VGG-16 [18] as the basic network to create a release element in this structure. This model is also based on Fast R-CNN. During training, we have many boxes with different sizes and different widths of objects throughout the image. The SSD detects a box with more Intersection-Over-Union (IoU) compared to ground reality. A detailed description of the structure of the SDD network layers is shown in [17].

Our main goal is to find a more robust model of various input sizes and shapes. Therefore, during SSD training a step was taken to maximize data. This process is made up of the following functions used for each image in the database:

- Use the whole original image to insert.

- Sample pool so that the minimum quantity of items is $0.1,0.3,0.5,0.7$, or 0.9 . The pixel size of each sample is an average of $[0.1,1]$ of the actual image

\section{Flow Chart:}

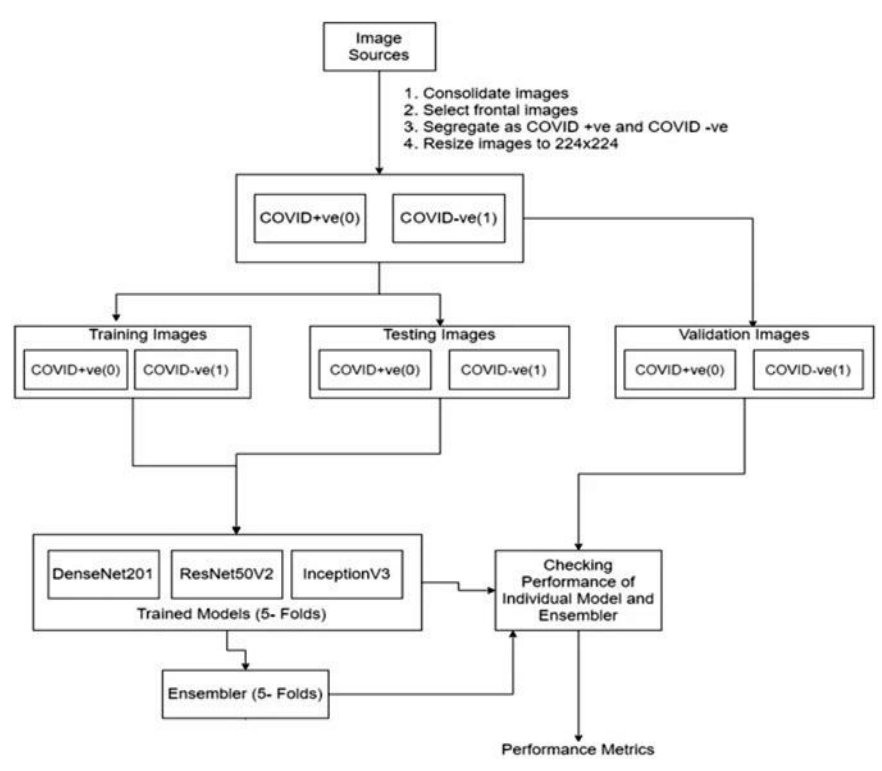

Figure 2 : Flow Chart of Our Approach 


\section{VISUALIZATIONS}

To illustrate the results correctly, we created our own speculative maps using RISE [6]. In this way, 1000 hidden versions from time to time of the given X-ray image are questioned and their classic scores are used to create a weighted mask corresponding to each output phase. The basic idea of the RISE method [6] is that masks that retain key parts of the image will result in higher creative points and hence the higher weight in the final screen of the appropriate category
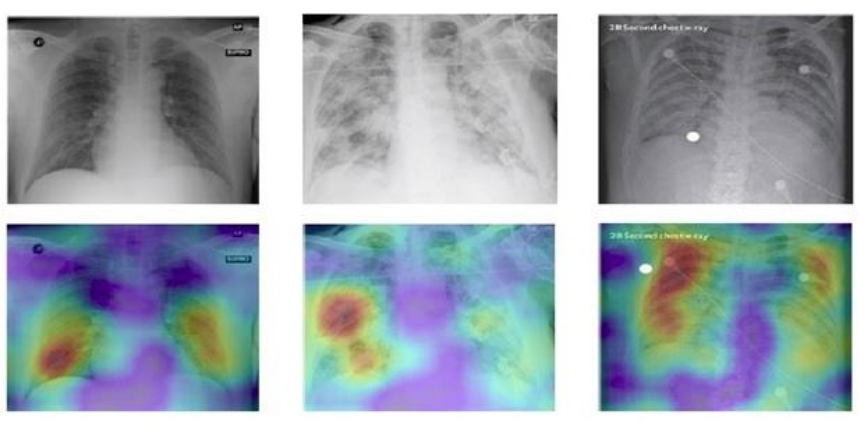

Figure 3. Saliency Map Visualization over Covid-19 Positive X-Rays.

The purpose of this demonstration was to have an additional check to limit the use of the model and to ensure that the focus regions were compliant with the relevant factors from the radiologist's point of view. Shows some saliency maps on COVID-19 X-rays.

\section{RESULT}

The final accuracy of the deep learning model is recorded as 0.98 and the loss is 0.04 . The deep learning model answers nearly the 0.98 of the chest $\mathrm{x}$ ray images of the patients correctly and, distinguishes between the infected and not infected lungs which are the first target region of human body.

Initial Result: The initial result shown by our model is listed below in form of training accuracy and validation accuracy

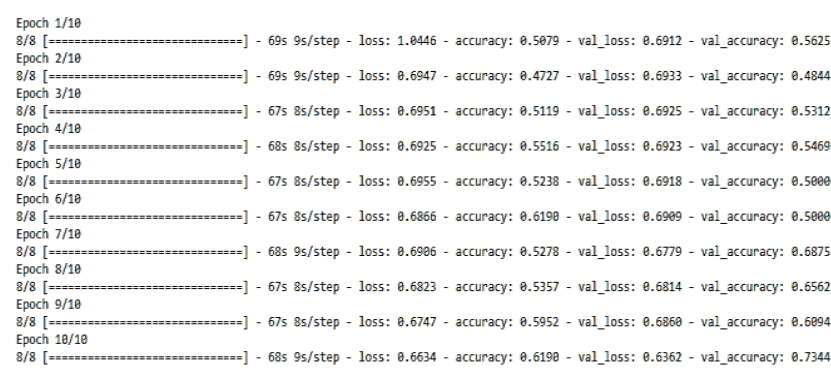

After Improvement : The initial result shown by our model is listed below in form of training accuracy and validation accuracy

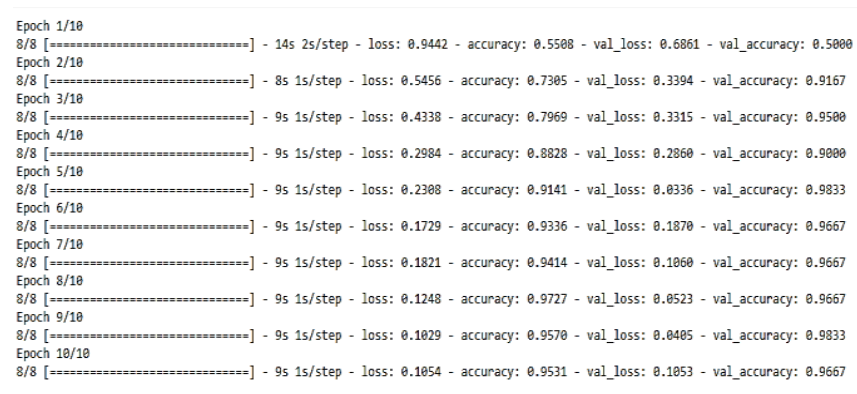

Figure 4. Results of initial and After Improvement

\section{CONCLUSION \& FUTURE PERSPECTIVE}

Adopting a three-pronged approach based on testing, classification and tracking of contacts is allowed to combat COVID-19. It is necessary to exploit the existing knowledge base to develop effective chemotherapeutic agents against COVID-19, taking clues from previous studies during other such emergencies. All areas ranging from surveillance and surveillance to prevention and treatment. As this is the third corona virus outbreak in recent times and many corona viruses circulating in animal ponds, we should focus on defining the pathway of SARS-CoV-2 cells and other corona viruses and increasing our preparedness by building capacity to prevent future outbreaks [67]. As the current situation necessitates the immediate delivery of solutions, the response to this emergence is greatly enhanced by various digital technologies and AI [68]. AI was found to be more consistent and accurate than human experts in finding COVID-19 and drug discovery. We need big data sets to train AI models and legal framework and ethics of data sharing before AI prioritizes testing and beyond. Several barriers to integrating AI with its full 
potential in the current environment are the discovery and sharing of clinical and disease information, computational resources, scalability, privacy and ethical concerns.

\section{REFERENCES}

[1]. Bennett, J.M.: Smart ct scan based covid19 virus detector. $\quad$ https: //github.com/JordanMicahBennett/SMART-CTSCAN BASED-COVID19 VIRUS DETECTOR (2020)

[2]. Cohen, J.P., Morrison, P., Dao, L.: Covid-19 image data collection. arXiv 2003.11597 (2020), https://github.com/ieee8023/covid-chestxraydataset

[3]. Huang, G., Liu, Z., van der Maaten, L., Weinberger, K.Q.: Densely connected convolutional networks. In: Proceedings of the IEEE Conference on Computer Vision and Pattern Recognition (2017)

[4]. Mooney, P.: Kaggle chest $\mathrm{x}$-ray images (pneumonia) dataset. https://www.kaggle. com/paultimothymooney/chest-xraypneumonia (2018)

[5]. Nisar,

https://github.com/zeeshannisar/covid-19.

https:/github.com/ zeeshannisar/COVID-19 (2020)

[6]. Petsiuk, V., Das, A., Saenko, K.: Rise: Randomized input sampling for explanation of black-box models. In: Proceedings of the British Machine Vision Conference (BMVC) (2018)

[7]. Rajpurkar, P., Irvin, J., Zhu, K., Yang, B., Mehta, H., Duan, T., Ding, D., Bagul, A., Langlotz, C., Shpanskaya, K., et al.: Chexnet: Radiologist-level pneumonia detection on chest $\mathrm{x}$-rays with deep learning. arXiv preprint arXiv:1711.05225 (2017)

[8]. Ranjan, E., Paul, S., Kapoor, S., Kar, A., Sethuraman, R., Sheet, D.: Jointly learning convolutional representations to compress radiological images and classify thoracic diseases in the compressed domain (12 2018). https://doi.org/10.1145/3293353.3293408

[9]. Ruiz, P.: Understanding and visualizing densenets. https://towardsdatascience. com/understanding-and-visualizing-densenets7f688092391a (2018)

[10]. Wang, L., Wong, A.: Covid-net: A tailored deep convolutional neural network design for detection of covid-19 cases from chest radiography images (2020)

[11]. Wang, X., Peng, Y., Lu, L., Lu, Z., Bagheri, M., Summers, R.M.: Chestx-ray8: Hospital-scale chest $\mathrm{x}$-ray database and benchmarks on weakly-supervised classification and localization of common thorax diseases. In: Proceedings of the IEEE conference on computer vision and pattern recognition. pp. 2097-2106 (2017)

[12]. Weng, X., Zhuang, N., Tian, J., Liu, Y.: Chexnet for classification and localization of thoracic diseases.

https:/github.com/arnoweng/CheXNet/ (2017) 13. Yao, L., Poblenz, E., Dagunts, D., Covington, B., Bernard, D., Lyman, K.: Learning to diagnose from scratch by exploiting dependencies among labels. arXiv preprint arXiv:1710.10501(2017)

\section{Cite this article as :}

Shashank Mishra, Himanshu Kumar Shukla, Rajiv Singh, Vivek Pandey, Shubham Sagar, Yasasvi Singh,

" Covid-19 Detection Using AI, International Journal of Scientific Research in Science, Engineering and Technology(IJSRSET), Print ISSN : 2395-1990, Online ISSN : 2394-4099, Volume 8, Issue 3, pp.561-566, May-June-2021. Available at doi $\quad$ : https://doi.org/10.32628/IJSRSET2183130 Journal URL : https://ijsrset.com/IJSRSET2183130 